\title{
Doktorarbeiten aus dem Medizinhistorischen Institut der Universität Zürich
}

a) Seit November 1968 gedruckte Dissertationen:

93. Dalcher R., Dr.Joh.Storchens, alias Pelargi. Theoretische und practische Abhandlung von Kinderkranckheiten, 1750/51 (zM A 59) 1968

94. Zehnder E., François Pourfour du Petit (1664-1741) und seine experimentelle Forschung über das Nervensystem (z M A 60) 1968

95. SсHönwetter H.P., Zur Vorgeschichte der Endokrinologie (z м A 61) 1968

96. Dickenmann W., Carl S.F.Credé (1819-1892) und seine Hauptleistungen (z м A 62) 1969

97. Rudolf H., Medizinische Chinoiserien, 1969

98. Pons G., Essai de Sociologie des Malades dans les Hôpitaux de Paris pendant les années 1815 à 1848 (z $\mathrm{mA} 63$ ) 1969

99. Zehnder O., Wilhelm Camerer (1842-1916) und der kindliche Stoffwechsel (т м A 64) 1969

100. Reнm K. E., Die Rolle des Buddhismus in der indischen Medizin und das Spitalproblem (z M A 65) 1969

b) Fertig, aber noch nicht gedruckt:

Keller, Die letzten Epidemien der suette miliaire

ROSNER, C.F. Heusinger

ACHaRD, Bidder

BrutTin, Hysterietheorien

Stinson, W.Lawrence, Surgeon

JAPP, Strümpell als Psychosomatiker

GuggenheIm, Bruno Bloch

Rich, E.H.Weber

Alder, Winslow

STEINEBRUNNER, Zürcher Spitalsarchitektur

WICK, Erismann

Kunz, Lavater

KESSLER, Folie à deux

WEBER, Moreau de Tours

WASER, Widerbelebung

MüLLER, Moritz Traube

ERLANGER, Leubuscher

von Felten, Döllinger's Pathologievorlesung

c) In Arbeit:

LEBRAM, Goll

BARDos, Hirnchirurgie in USA 
Eichmann, Ismar Boas

Eidenbenz, Heiliggeistspitäler

KraEgel, Serres

Cellier, Buchheim

Muheim, Severino

R.MüLLER, Josef Frank

StäUbl, Phobien

Тнӧму, Herzkrankheiten im 17. Jahrhundert

Stahel, Der weiße Mantel

Rutz, Die Garrods

WAGNER, Hitzig

LuZI, Graubündner Gesetze

d) Neu dazugekommen:

HürzeleR, Whytt

Ullmann, Sylvat. Gelbfieber

Rosenberg, Tabes

EgLI, Prix Monthyon

KNüsel II, Luzerner Bäder

EKERT, L'ennui

Rido, Moritz Schiff

Hess, Cohnheim

Bolliger, Schlaftheorien

KNÜsel I, Luzerner Prüfungen 